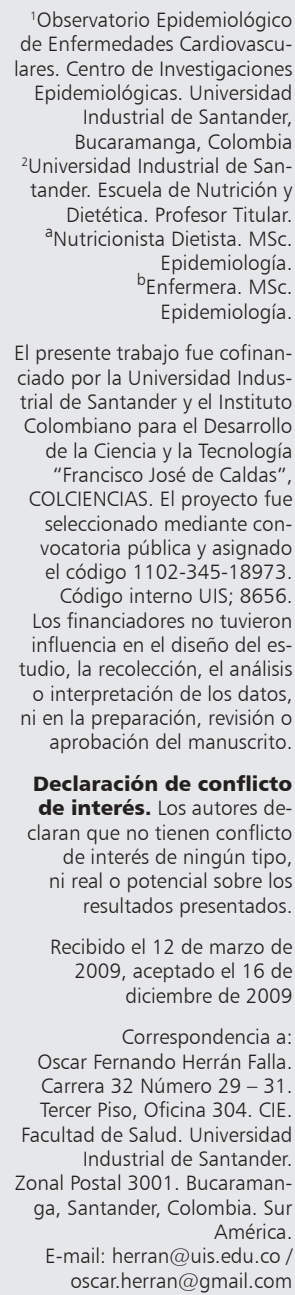

'Observatorio Epidemiológico de Enfermedades Cardiovasculares. Centro de Investigaciones Epidemiológicas. Universidad Industrial de Santander Bucaramanga, Colombia ${ }^{2}$ Universidad Industrial de Santander. Escuela de Nutrición y Dietética. Profesor Titular. ${ }^{a}$ Nutricionista Dietista. MSC. Epidemiología. ${ }^{b}$ Enfermera. MSc Epidemiología.

El presente trabajo fue cofinanciado por la Universidad Industrial de Santander y el Instituto Colombiano para el Desarrollo de la Ciencia y la Tecnología "Francisco José de Caldas",

COLCIENCIAS. El proyecto fue seleccionado mediante convocatoria pública y asignado el código 1102-345-18973. Código interno UIS; 8656 Los financiadores no tuvieron influencia en el diseño del estudio, la recolección, el análisis o interpretación de los datos, ni en la preparación, revisión o aprobación del manuscrito.

Declaración de conflicto de interés. Los autores declaran que no tienen conflicto de interés de ningún tipo

ni real o potencial sobre los resultados presentados.

Recibido el 12 de marzo de 2009, aceptado el 16 de diciembre de 2009 Correspondencia a: Oscar Fernando Herrán Falla. Carrera 32 Número 29 - 31 Tercer Piso, Oficina 304. CIE. Facultad de Salud. Universidad Industrial de Santander. Zonal Postal 3001. Bucaramanga, Santander, Colombia. Sur América.

E-mail: herran@uis.edu.co / oscar.herran@gmail.com

\section{Análisis confirmatorio para la prueba AEQ-III en Bucaramanga, Colombia}

\author{
OSCAR F. HERRÁN ${ }^{1,2, a}$, MARÍA F. ARDILA ${ }^{1, b}$.
}

\section{Confirmatory analysis for the test AEQ-III in Bucaramanga, Colombia}

\begin{abstract}
Background: The Alcohol Expectancy Questionnaire-III (AEQ-III) test evaluates positive expectations towards alcohol consumption. Aim: To perform a confirmatory factorial analysis of the AEQ-III test. Material and Methods: During 2006-2008, 1199 adults between 18 and 60 years old (588 males) answered a survey that included socio-economic variables and the AEQ-III test to determine alcohol consumption and its consequences. A factorial analysis was performed and the goodness of fit of the final model was assessed. Results: "Increase in expressivity and sexuality", "decrease in physical tension", "lack of inhibition and feelings of power" and "decrease in psychological tension" were the four confirmed dimensions. This model differs of others applied in the region, has 22 variables and adjusts well. Values for median quadratic approximation error, Tucker-Lewis index, normal adjustment index and goodness of fit were 0.07, 0.93 and 0.92, respectively. The variance explained by the model increased from $62 \%$ in the exploratory to $76 \%$ in the confirmatory analysis. Internal consistency in the exploratory and confirmatory models was 0.85 and 0.87 . Conclusions: Two confirmed dimensions correspond to group behaviors and the others, to individual behaviors. All indices of relevance of grouping variables, goodness of fit and parsimony were maintained and even improved and are generally optimal.

(Rev Med Chile 2010; 138: 88-94).
\end{abstract}

Key words: Alcoholism; Colombia; Primary prevention.
L a teoría de las expectativas ha sido en las dos últimas décadas, la más útil para explicar el consumo de alcohol, el consumo problemático y el alcoholismo ${ }^{1-9}$. Las seis expectativas propuestas en 1985, fueron derivadas del análisis factorial de la prueba Alcohol Expectancy Questionnaire (AEQ-III) ${ }^{10}$. AEQ-III evalúa las esperanzas o expectativas que determinan el consumo de alcohol y mide el grado en que un individuo espera una variedad de efectos generales y específicos al consumir alcohol ${ }^{7,10}$. Esta prueba ha sido utilizada en Centro y Sur América para explicar con base en la teoría de las expectativas, el consumo de alcohol y sus consecuencias en la población general y en estudiantes universitarios ${ }^{4,5,11-16}$.
A pesar de la variedad de las intervenciones clínicas y poblacionales, del endurecimiento de la legislación, de los impuestos que incrementan el valor de las bebidas alcohólicas para desestimular su consumo, y de prohibiciones explicitas, en Bucaramanga el consumo de alcohol y sus consecuencias indeseables siguen aumentando ${ }^{17}$. Estudios locales y las encuestas nacionales evidencian que más de $90 \%$ de la población adulta consume alcohol y 14\% según la prueba CAGE es alcohóli$\mathrm{Ca}^{17-19}$. El consumo de alcohol genera alta carga de mortalidad, morbilidad y gasto en salud ${ }^{17-21}$. El estudio de los posibles determinantes del consumo, el consumo problemático y la dependencia cobra relevancia en la medida en que en esta población 
ninguna variable biológica, sociodemográfica o dietaria esta asociada a la ingesta de alcohol ${ }^{15,17,22}$.

La utilización de cualquier prueba de medición desarrollada en contextos socioculturales incluso similares, requiere además de adaptarla al lenguaje local, de estudios de reproducibilidad y validez ${ }^{23,24}$. El estudio de pruebas predesarrolladas como el AEQ-III pertenece al campo de la evaluación de la tecnología diagnóstica, el cual debe desarrollarse con urgencia en los países en vía de desarrollo ${ }^{24}$.

Este estudio tuvo como objetivos; conducir el análisis factorial confirmatorio de un modelo con cuatro factores derivado previamente de la prueba AEQ-III en población adulta y joven de Bucaramanga, Colombia ${ }^{8}$. Además, comparar el modelo diseñado con el originalmente propuesto en 1985 y otro desarrollado en 2000 y que ha influenciado positivamente el estudio del consumo de alcohol en América Latina ${ }^{3-5}$.

\section{Material y Método}

Bucaramanga es una ciudad intermedia en la región nororiental de Colombia, su actividad económica es el comercio, cuenta con 900.000 habitantes, y de clima cálido, en promedio $26^{\circ} \mathrm{C}$. Durante el periodo 2006 a 2008 se desarrolló un estudio analítico de validez factorial.

Tamaño de la muestra y selección de sujetos. Mil ciento noventa y nueve sujetos fueron encuestados. El tamaño muestral fue definido como diez por el número de ítems del AEQ-III (10 x 120). Diez es el número recomendado de sujetos por ítem para establecer la validez factorial de un cuestionario $^{25,26}$. Los sujetos fueron seleccionados a través de muestreo en múltiples etapas para representar por sexo y estrato socioeconómico a la población. Los que cumplieron los siguientes criterios fueron elegibles; 1) Entre 18 y 60 años; 2) Residir en la ciudad dos años o más; 3) No asistir a un grupo de apoyo como alcohólicos anónimos y 4) Que manifestaran su deseo de participar suministrando voluntariamente la información. De un mapa de Bucaramanga fueron elegidas al azar manzanas y luego casas por estrato socioeconómico, en cada vivienda se realizó un censo de elegibles y aleatoriamente se seleccionó uno por vivienda. Si esta persona se rehusaba, se elegía otra persona de la misma vivienda. Después de seleccionarlos, de informarlos sobre los objetivos, de garantizar la confidencialidad y de obtener un consentimiento escrito, se encuestaron. El proyecto y los procedimientos fueron aprobados por el Comité de Ética en Investigación de Salud de la Universidad Industrial de Santander.

Fuentes de información. Se aplicó un cuestionario sobre aspectos sociodemográficos y el cuestionario AEQ-III ${ }^{8,10}$. Los procedimientos para adaptar el cuestionario AEQ-III al idioma y jerga de la población fueron descritos previamente ${ }^{8}$.

Estadística. El análisis factorial confirmatorio se dividió en tres etapas, en dos de ellas se replicaron modelos desarrollados previamente ${ }^{4,8}$ y en la otra se desarrolló uno. Para todos los modelos se evaluaron las soluciones factoriales y la bondad de ajuste. En la primera etapa se replicó el Modelo A con cuatro factores, para el total de la muestra y en la población menor de 25 años $^{8,15}$. La segunda etapa estableció una solución factorial con seis factores; Modelo B ${ }^{3,10}$. La tercera etapa replicó la solución factorial con ocho ítems desarrollada para estudiantes universitarios mexicanos; Modelo $C^{4,5}$. La agrupación de variables estaba predeterminada para los modelos con cuatro y ocho factores, no para el modelo de seis. Los criterios para la selección y agrupación de variables en el Modelo $B$ fueron: la mayor carga factorial de la variable al factor, un valor de carga mínimo de 0,40 y complementariamente una varianza compartida o común entre variables mínima de 0,40 (1 - Uniqueness). En todas las soluciones factoriales se utilizó una rotación PROMAX debido a la correlación en los factores latentes ${ }^{27}$.

Para evaluar la pertinencia de la agrupación de variables dentro de factores, se calculó el porcentaje de la varianza explicada y el estadístico Kaiser-Meyer-Olkin (KMO), tanto para las variables como para los factores ${ }^{27}$.

Con el fin de confirmar el modelo con cuatro factores en la muestra y los menores de 25 años, $y$ de permitir su comparación con el de seis y ocho factores, fueron calculados índices de bondad de ajuste; razón de verosimilitud $\left(\chi^{2}\right)$, error de aproximación cuadrático medio (RMSEA), el índice Tucker-Lewis (NNFI), de ajuste normal (NFI), de bondad de ajuste (GFI) y el índice de ajuste de parsimonia (PNFI) ${ }^{27}$. Todas las soluciones factoriales, fueron realizadas con la matriz de covarianza y la estimación de la máxima verosimilitud ${ }^{27-29}$. Para cada modelo la consistencia interna intra factor y entre factores fue determinada con el alfa de 
Cronbach $(\mathrm{AC})^{18,30}$. El coeficiente rho de Spearman permitió establecer la correlación entre factores. Los puntajes en los factores fueron escalas aditivas.

La comparación entre los puntajes alcanzados en las expectativas y algunas variables como la edad, el sexo o la escolaridad, se realizó con base en medias ajustadas a través de regresión lineal y utilizando pruebas $t$ de Student y ANOVA. Toda la información fue sometida a doble digitación y validada en EpiInfo v 6.04d $\mathrm{d}^{31}$. La descripción de las variables sociodemográficas y los puntajes alcanzados en las expectativas fue realizada con medidas descriptivas apropiadas e intervalos de confianza del 95\% (IC). Los estratos socioeconómicos 1 y 2, los más bajos en la escala de ingreso y desarrollo, se agruparon en el nivel socioeconómico 1, los 3 y 4 en el nivel 2 y los estratos 5 y 6 en el nivel 3. Dada la aplicación de un diseño de muestreo en múltiples etapas, la estimación de los errores se realizó utilizando el ajuste de la varianza de Taylor ${ }^{32,33}$. El procesamiento de las variables y los cálculos estadísticos, fueron realizados con STATA/SE versión $10^{34}$. Los MEE fueron realizados con $\mathrm{BDMP}^{35}$.

\section{Resultados}

Población estudiada. Un total de 3.562 casas fueron visitadas, 4.940 sujetos fueron declarados elegibles, de ellos 1.199 se encuestaron. Entre 195 y 206 sujetos participaron por cada uno de los seis estratos socioeconómicos originales. El 49\% eran hombres, la edad media en la muestra fue de 35,9 años (IC: 35,2 a 36,6) sin diferencia por sexo $\mathrm{p}=0,227$. El 4,8\% no tenía educación primaria. La escolaridad media era de 11,5 años (IC: 11,3 a 11,8 ), un año mayor en las mujeres $\mathrm{p}=0,003$. $\mathrm{La}$ escolaridad estaba inversamente relacionada con el nivel socioeconómico $\mathrm{p}=0,000$. Otras características de la población estudiada se presentan en la Tabla 1.

Tabla 1. Características de la población estudiada

\begin{tabular}{|c|c|c|c|}
\hline Variable & $\begin{array}{c}\text { Total } \\
(n=1.199)\end{array}$ & $\begin{array}{l}\text { Hombre } \\
(n=588)\end{array}$ & $\begin{array}{c}\text { Mujer } \\
(n=611)\end{array}$ \\
\hline Ingesta de alcohol/semana $(\mathrm{g})$ * & $54,8(48,4$ a 61,2$) \dagger$ & $83,3(71,9$ a 94,7$)$ & $27,3(22,0$ a 32,5$)$ \\
\hline \multicolumn{4}{|l|}{ Escolaridad (años) * } \\
\hline Hasta primaria & $188\{15,7\}$ & $80\{13,6\}$ & $108\{17,7\}$ \\
\hline Entre 6 y 11 & $441\{36,8\}$ & $200\{34,0\}$ & $241\{39,4\}$ \\
\hline Entre 12 y 16 & $444\{37,0\}$ & $244\{41,5\}$ & $200\{32,7\}$ \\
\hline 17 ó más & $126\{10,5\}$ & $64\{10,9\}$ & $62\{10,2\}$ \\
\hline \multicolumn{4}{|l|}{ Nivel socioeconómico ‡ } \\
\hline Uno & $399\{33,3\}$ & $188\{32,0\}$ & $211\{34,5\}$ \\
\hline Dos & $407\{33,9\}$ & $201\{34,2\}$ & $206\{33,7\}$ \\
\hline Tres & $393\{32,8\}$ & $199\{33,8\}$ & $194\{31,8\}$ \\
\hline \multicolumn{4}{|l|}{ Puntaje en las expectativas $₫$} \\
\hline Incremento de expresividad y sexualidad & $2,0 \quad(1,9$ a 2,1$)$ & $2,0 \quad(1,9$ a 2,1$)$ & $1,9(1,8$ a 2,0$)$ \\
\hline Disminución de la tensión física & $1,7 \quad(1,7$ a 1,8$)$ & $1,8 \quad(1,6$ a 1,9$)$ & $1,6(1,5$ a 1,8$)$ \\
\hline Desinhibición y sentimientos de poder & $4,8 \quad(4,8$ a 4,9$)$ & $4,9 \quad(4,7$ a 5,1$)$ & $4,7(4,6$ a 4,9$)$ \\
\hline Disminución de la tensión psicológica * & $1,9 \quad(1,9$ a 2,0$)$ & $2,0 \quad(1,8$ a 2,1$)$ & $1,8(1,6$ a 1,9$)$ \\
\hline Sumatoria total & $10,5(10,4$ a 10,7$)$ & $10,8(10,3$ a 11,2$)$ & $10,2(9,6$ a 10,8$)$ \\
\hline
\end{tabular}

${ }^{*} p<0,05$. † Promedio e intervalo de confianza del 95\%. | Número y porcentaje. $\ddagger$ Al nivel uno pertenecen los estratos socioeconómicos 1 y 2; al dos, los estratos 3 y 4 ; al nivel tres, pertenecen los estratos 5 y 6 . 9 Promedio e intervalo de confianza del 95\%, ajustado por la edad, la escolaridad, el nivel socioeconómico, la cantidad de alcohol consumida (g) y la clasificación alcanzada en CAGE y CP-alcohol. 
Análisis factorial confirmatorio. El número de variables constitutivas del modelo con cuatro factores: Modelo A, es substancialmente menor que el número de ellas en el modelo clásico de seis factores, Modelo B, y el desarrollado en México con ocho factores, Modelo C. A pesar de esto, la pertinencia de la agrupación en el Modelo A es relativamente superior de acuerdo con el estadístico $\mathrm{KMO} ; 0,82,0,86$ y 0,87 respectivamente (Tabla 2 ). En términos de parsimonia o del balance entre el porcentaje de la varianza explicada y el número de variables, el Modelo A es muy superior; mientras que con 22 variables se logró explicar el 76\% del to- tal de la variabilidad, para obtener un aumento del $9 \%$ se requieren 29 variables adicionales (Tabla 2 ).

Un total de diez factores fueron identificados en los tres modelos. El Modelo A, tiene tres de los seis del Modelo B y dos del Modelo C (Tabla 3). La consistencia interna según el AC en el Modelo A estuvo entre 0,62 y 0,74 , en el Modelo B entre 0,75 y 0,93 , en el Modelo C entre 0,48 y 0,86 . El AC para los tres modelos puede considerarse como muy bueno (Tabla 3). La correlación entre factores en el Modelo A varió entre 0,47 y 0,69. La rho de Spearman entre "Incremento de la expresividad y la sexualidad" y "Disminución de la tensión física"

Tabla 2. Pertinencia de la agrupación de variables y del número de factores

\begin{tabular}{|lccccc|}
\hline $\begin{array}{l}\text { Modelo } \\
\text { (Factores) }\end{array}$ & $\begin{array}{c}\text { Varianza (\%) } \\
\text { Explicada }\end{array}$ & $\begin{array}{c}\text { Número } \\
\text { Número }\end{array}$ & $\begin{array}{c}\text { Estadístico } \\
\text { KMO * }\end{array}$ & $\begin{array}{c}\text { Estadístico } \\
\text { KMO * } \mathbf{~}\end{array}$ & Diferencia \\
\hline Modelo A (4) & 76 & 22 & 0,98 & 0,82 & \\
Modelo B (6) & 82 & 46 & 0,98 & 0,86 & 0,04 \\
Modelo C (8) & 85 & 51 & 0,98 & 0,87 & 0,05 \\
\hline
\end{tabular}

* Est. $\mathrm{KMO}^{27}$. Estadístico Kaiser-Meyer-Olkin para la pertinencia de la agrupación; para el número de variables seleccionadas y para el número de factores. † Para las variables. ‡ Para los factores.

Modelo $A^{8,15}$; Modelo confirmatorio en adultos y jóvenes de Bucaramanga, Colombia.

Modelo $\mathrm{B}^{3,10}$; Modelo clásico con seis factores.

Modelo $\mathrm{C}^{4,5}$; Modelo desarrollado en estudiantes universitarios mexicanos, y replicado en la muestra.

Tabla 3. Consistencia interna* para las expectativas positivas hacia el consumo, en tres modelos

\begin{tabular}{|c|c|c|c|}
\hline $\begin{array}{l}\text { No. de Factores (No. de Variables) } \\
\text { Expectativa }\end{array}$ & $\begin{array}{c}\text { Modelo A } \\
4(22)\end{array}$ & $\begin{array}{c}\text { Modelo B } \\
6(46)\end{array}$ & $\begin{array}{l}\text { Modelo C } \\
8(51)\end{array}$ \\
\hline Incremento de la expresividad y la sexualidad & 0,62 & & \\
\hline Desinhibición y sentimientos de poder & 0,68 & 0,75 & \\
\hline Agresividad y sentimientos de poder & & & 0,86 \\
\hline Cambios psicofisiológicos & & & 0,48 \\
\hline Desinhibición & & & 0,68 \\
\hline Disminución de la tensión física & 0,66 & 0,86 & 0,67 \\
\hline Disminución de la tensión psicológica & 0,74 & 0,93 & 0,69 \\
\hline Expresividad verbal & & 0,76 & 0,78 \\
\hline Incremento de la sexualidad & & 0,87 & 0,81 \\
\hline Interacción grupal & & 0,85 & 0,85 \\
\hline Para el conjunto de variables & 0,87 & 0,96 & 0,95 \\
\hline
\end{tabular}

Para todos los modelos $n=1.199$. *Alfa de Cronbach.

Modelo $A^{8,15}$; Modelo confirmatorio en adultos y jóvenes de Bucaramanga, Colombia.

Modelo $B^{3,10}$; Modelo clásico con seis factores.

Modelo C4,5; Modelo desarrollado en estudiantes universitarios mexicanos, y replicado en la muestra. 
Análisis confirmatorio para la prueba AEQ-III en Bucaramanga, Colombia - O. F. Herrán y M. F. Ardila

Tabla 4. Análisis de factores: Medidas de bondad de ajuste para los tres modelos evaluados

\begin{tabular}{|c|c|c|c|}
\hline $\begin{array}{l}\text { No. de Factores (No. de Variables) } \\
\text { Índice }\end{array}$ & $\begin{array}{l}\text { Modelo A } \\
4 \text { (22) }\end{array}$ & $\begin{array}{l}\text { Modelo B } \\
6(46)\end{array}$ & $\begin{array}{l}\text { Modelo C } \\
8 \text { (51) }\end{array}$ \\
\hline Razón de verosimilitud $\left(\chi^{2}\right)^{*}$ & $1.147(183) \dagger$ & $4.854(973)$ & $4.857(974)$ \\
\hline Error de aprox. cuadrático medio (RMSEA) & 0,07 & 0,06 & 0,06 \\
\hline Índice Tucker-Lewis (NNFI) & 0,93 & 0,98 & 0,98 \\
\hline Índice de ajuste Normal (NFI) & 0,93 & 0,97 & 0,97 \\
\hline Índice de bondad de ajuste (GFI) & 0,92 & 0,85 & 0,85 \\
\hline Índice de ajuste de parsimonia (PNFI) & 0,81 & 0,91 & 0,91 \\
\hline
\end{tabular}

Para todos los modelos $n=1.199$. * Para todos valor de $p<0,05$. $†$ Valor de $\chi^{2}$ y grados de libertad.

Modelo $A^{8,15}$; Modelo confirmatorio en adultos y jóvenes de Bucaramanga, Colombia.

Modelo $\mathrm{B}^{3,10}$; Modelo clásico con seis factores.

Modelo $C^{4,5}$; Modelo desarrollado en estudiantes universitarios mexicanos, y replicado en la muestra.

fue de 0,53; entre "Incremento de la expresividad y la sexualidad" y "Desinhibición y sentimientos de poder" de 0,66; entre "Incremento de la expresividad y la sexualidad" " "Disminución de la tensión psicológica" de 0,69; entre "Disminución de la tensión física" y "Desinhibición y sentimientos de poder" de 0,47; entre "Disminución de la tensión física" y "Disminución de la tensión psicológica" de 0,59 y entre "Desinhibición y sentimientos de poder" $y$ "Disminución de la tensión psicológica" de $0,64, p<0,001$ para todas las correlaciones.

La razón de verosimilitud en el Modelo A para menores de 25 años fue de 390, con 183 grados de libertad, en el total de la muestra de 1147 con 183 grados de libertad. Complementariamente, en los menores de 25 años el RMSEA fue de 0,06, el NNFI de 0,93, el NFI de 0,89, la bondad de ajuste GFI de 0,91 y el índice de ajuste de parsimonia PNFI 0,77. El Modelo A para el total de la muestra de acuerdo con la razón de verosimilitud $\left(\chi^{2}\right)$ es equivalente al de menores de 25 años. Los Modelos $\mathrm{B}$ y C de acuerdo con el valor de $\chi^{2}$ son diferentes del Modelo A, pero equivalentes entre ellos. Para todos los Modelos los índices de bondad de ajuste son muy buenos (Tabla 4).

\section{Discusión}

Limitaciones y alcances del estudio. Debido al diseño, los resultados son aplicables a mayores de 18 años con el patrón de consumo y la cultura hacia el alcohol en Bucaramanga ${ }^{15,17,18,22,23}$. La dis- tribución por sexo y la proporción de primaria incompleta son similares a las reportadas en la encuesta de demografía y salud ${ }^{36}$, de acuerdo con lo anterior, la muestra representó adecuadamente a la población blanco. El estudio de las expectativas cobra relevancia dado que han demostrado su utilidad en la prevención primaria, secundaria y en el tratamiento del consumo problemático y la dependencia ${ }^{38,43}$.

Análisis confirmatorio. La varianza explicada por el Modelo A paso de $62 \%$ en el análisis exploratorio a $76 \%$ en el confirmatorio, la consistencia interna en el modelo exploratorio fue de 0,85 , en el confirmado de $0,87^{8}$. Todos los índices de pertinencia de agrupación de variables, bondad de ajuste y parsimonia mejoraron, y en general son óptimos ${ }^{27}$. Un valor ideal de $\chi^{2}$ debe estar por debajo de 0,08, en el Modelo A fue de 0,07. Para los demás índices el valor ideal debe ser cercano a uno como en efecto se demostró ${ }^{27}$. El modelo A presenta ventajas relativas frente a los Modelos B y C; en términos de parsimonia explica $76 \%$ de la varianza con sólo 22 variables, mientras que el Modelo B explica el 82\% con 46, y el Modelo C $85 \%$ con 51 variables, en segundo lugar los índices de bondad de ajuste son equivalentes, en tercero, las dimensiones identificadas aún acordes con la teoría se agrupan de manera diferente, y esto justifica la importancia de evaluar la tecnología diagnóstica, pues las futuras intervenciones serán diseñadas acordes "a la medida" del contexto. El aprendizaje social y la generación cultural de expectativas positivas hacia el consumo de alcohol 
en Bucaramanga, Colombia, sólo comparte tres factores con el modelo propuesto originalmente de seis; "Desinhibición y sentimientos de poder", "Disminución de la tensión física" y "Disminución de la tensión psicológica" ${ }^{3,10}$, y sólo dos factores con el desarrollado en estudiantes mexicanos; "Disminución de la tensión física" y "Disminución de la tensión psicológica" ${ }^{4,5}$. El 50\% de los factores en el Modelo A se relacionan con el comportamiento grupal, el 50\% restante con el individual, en el Modelo B y en C, el $67 \%$ y $62 \%$ de los factores respectivamente, están relacionados con el comportamiento grupal. Contrario a la evidencia, el sexo no está asociado al puntaje total alcanzado en las expectativas, tampoco con ninguno de los cuatro factores excepto para el factor "Disminución de la tensión psicológica"; factor con puntajes mayores en los hombres, mientras que en la literatura se reporta para las mujeres ${ }^{4-6,8,14-16}$. En Perú, se informó de una solución factorial derivada de la versión AEQ-A que explica 27,8\% de la varianza a través de tres factores ${ }^{12}$. Lo anterior permite intuir variantes en la cultura que necesariamente deben incorporarse en las estrategias de intervención basadas en el AEQ-III, so pena de obtener resultados inválidos o contradictorios ${ }^{43}$.

Utilidad de los resultados. Las expectativas identificadas en población joven y adulta son reproducidas por los menores de edad y los escolares como parte del proyecto cultural de las poblaciones ${ }^{15}$. En esta población y otras, a los doce años cualquier sujeto ha estado expuesto racionalmente a experiencias directas o indirectas relacionadas con el consumo de alcohol, el consumo problemático y el alcoholismo ${ }^{6-8}$; por lo anterior, es posible con base en las cuatro dimensiones identificadas en el Modelo A, diseñar e implementar intervenciones en edades tempranas. Hay evidencia de que al tratar en escolares o en edades aún más tempranas aspectos relacionados con las expectativas, incluso sin llegar a modificarlas, se puede retardar la edad de inicio en el consumo de alcohol ${ }^{6,7,17}$, aspecto relevante, ya que en esta población los sujetos con mayores edades de inicio en el consumo de alcohol, retardan la de la primera embriaguez y disminuyen substancialmente su riesgo de consumo problemático y alcoholismo ${ }^{17}$. Las expectativas también han sido relacionadas como predictoras de la cantidad de alcohol consumida y de éxito en el tratamiento de consumidores problemáticos y alcohólicos, altos puntajes en las expectativas, han sido relacionados con mejores resultados en el tratamiento y a su vez, mejores resultados en el tratamiento se han relacionado con disminución de las expectativas $2,7,9,14,37-40$.

Aún hay poca evidencia sobre relaciones causales entre expectativas y consumo de alcohol, sin embargo, el estudio de las motivaciones hacia el consumo y dentro de ellas el de las expectativas, es una oportunidad para aprender más desde las neurociencias sobre los aspectos cognitivos relacionados con el consumo de alcohol. Es necesario evaluar el impacto de las intervenciones basadas en las expectativas positivas frente a otras ya establecidas, además de evaluar en qué grupos de edad éstas son más efectivas ${ }^{7}$, y estudiar los moderadores de las expectativas, pues los resultados aún son contradictorios ${ }^{2,7,37-43}$.

Finalmente, los resultados presentados permitirán con mucha confianza diseñar, implementar y evaluar estrategias de intervención primaria y secundaria en diferentes grupos de edad, estudiar los moderadores de las expectativas positivas hacia el CA, como las ventajas relativas de utilizar esta teoría frente a otras para la prevención del consumo de alcohol, sus consecuencias negativas y la cantidad de alcohol consumida.

\section{Referencias}

1. Brown SA, Goldman MS, Inn A, Anderson LR. Expectactions of reinforcement from alcohol their domain and relation to drinking patterns. J Consult Clin Psychol 1980; 48: 419-26.

2. Oei TP, Baldwin AR. Expectancy theory: a two-process model of alcohol use and abuse. J Stud Alcohol 1994; 55 : 525-34.

3. Goldman MS, Del Boca FK, Darles J. Alcohol expectancy theory: The application of cognitive neuroscience. En: Leonard KE, Blane HT (Editors). Psychological theories of drinking and alcoholism. Second Edition. New York: Guilford Press 1999: 203-46.

4. Mora-Ríos J, Natera G, Villatorio J, Villalvazo R. Validez factorial del cuestionario de expectativas hacia el alcohol (AEQ-III) en estudiantes universitarios. Psicología Conductual. 2000; 8: 319-28.

5. Mora J, Natera G. Expectativas, consumo de alcohol y problemas asociados en estudiantes universitarios de la ciudad de México. Salud Publica Mex 2001; 43: 89-96.

6. Jones BT, Corbin W, Fromme K. A review of expectancy theory and alcohol Consumption. Addiction 2001; 96: 57-72.

7. Leigh BC, Stacy AW. Alcohol expectancies and drinking in different age groups. Addiction. 2004; 99: 215-27.

8. Herrán OF, Ardila MF. Validez factorial del cuestionario 
de expectativas hacia el consumo de alcohol (AEQ-III), en adultos de Bucaramanga, Colombia. Rev Med Chile 2007; 135: 488-95.

9. Wall AM, Thrussell C, Lalonde RN. Do alcohol expectancies become intoxicated outcomes? A test of sociallearning theory in a naturalistic bar setting. Addictive Behav 2003; 28: 1271-83.

10. Brown SA, Christiansen BA, Goldman MS. The alcohol expectancy questionnaire: an instrument for the assessment of adolescent and adult alcohol expectancies. J Stud Alcohol.1987; 48: 483-91.

11. Oliveira MS, Werlang BG. Inventário de expectativas e crenças pessoais acerca do álcool (IECPA) - Versão brasileira adaptada. São Paulo: Casa do Psicólogo; 1993.

12. Pérez-Aranibar, CC, Van den Broucke S, Fontaine J. Validation of the alcohol expectancy questionnaire (AEQ-A) for Peruvian university students. J Alcohol Drug Educ 2005; 49: 63-85.

13. Acero IC. Consumo de alcohol en universitarios: Relación funcional con los factores Sociodemográficos, Las expectativas y la ansiedad social. Act.Colom.Psicol 2005; 13: 91-119.

14. Mora-Ríos J, Natera G, Juarez F. Expectativas relacionadas con el alcohol en la predicción del abuso en el consumo en jóvenes. Salud Mental 2005; 8: 82-90.

15. Ardila MF, Herrán OF. Expectativas en el consumo de alcohol en Bucaramanga, Colombia. Rev Med Chile 2008; 136: 73-82.

16. Londoño-Pérez C, García-Hernández W, Valencia-Lara SC, Vinaccia-Alpi S. Expectativas frente al consumo de alcohol en jóvenes universitarios colombianos. Anales de Psicología. 2005; 21: 259-67.

17. Herrán OF, Ardila MF. Consumo de alcohol, riesgo de alcoholismo y alcoholismo en Bucaramanga, Colombia, 2002-2003. Colomb Med 2005; 36: 158-67.

18. Herrán OF, Ardila MF. Validez interna y reproducibilidad de la prueba CAGE en Bucaramanga, Colombia. Biomédica 2005; 25: 231-41.

19. Presidencia de la Republica/Ministerio de Salud/Programa Rumbos. Estudio nacional sobre uso y abuso de alcohol y consumo de sustancias psicoactivas, Colombia 2000.

20. Miguez UH. Epidemiología de la alcoholización juvenil en Argentina. Acta psiquiátr psicol Am Lat 2004; 50: 43 7.

21. Rehm J, Monteiro M. Alcohol consumption and burden of disease in the Americas: implications for alcohol policy. Rev Panam Salud Pública 2005; 18: 241-8.

22. Herrán OF, Ardila MF, Barba DM. Consumo problemático de alcohol en Bucaramanga, Colombia: diseño y validación de una prueba. Biomédica 2008; 28: 25-37.

23. Validity and reproducibility of two semi-quantitative alcohol frequency questionnaires for the Colombian population. Public Health Nutr 2006; 6: 763-70.

24. Kraemer HC. Evaluating Medical Tests. London: Sage Publications, 1992; 63-95.

25. Norman G, Streiner D. Bioestadística. Barcelona: Edito- rial Mosby 1996; 129-48.

26. Tavella NM. Análisis de los itemes en la construcción de instrumentos psicométricos. México: Editorial Trillas $1978 ; 75$.

27. Hair JF, Anderson RE, Tatham RL, Black WC. Análisis multivariante. Quinta edición, Madrid: Prentice Hall, 1999.

28. Hamilton LC. Regression with graphics. Belmont, California: Duxbury Press 1991; 249-88.

29. Kline P. An easy guide to factor analysis. London: Routledge Press 1994; 157-84.

30. Gliem JA, Gliem RR. Calculating, interpreting, and reporting Cronbach's alpha coefficient for Likert type scales. Presented at the Midwest research to practice conference in adult, continuing, and community education. The Ohio State University, Columbus, $\mathrm{OH}$, October 8-10, 2003. Disponible en; www.alumni-osu.org/midwest/midwest $\% 20$ papers/Gliem\%20\&\%20Gliem--Done. pdf [Acceso el 23 de Marzo de 2008].

31. CDC. EpiInfo, versión 6.04d. Epidemiología en ordenadores. Atlanta, Georgia. Enero, 2001.

32. STATA. Estimation of means, totals, ratios, and proportions for survey data. STB 1996 ;6: 213-35.

33. Levy SP, Lemeshow S. Sampling of populations: Methods and applications. 3th ed. New York: John Wiley \& Sons; 1999: 365-90.

34. StataCorp, 2007. Stata Statistical Software: Release 10. Collegue Station, TX: StataCorp LP.

35. BDMP, 1992. Statistical Software Inc: Release 7.

36. PROFAMILIA. Encuesta Nacional de Demografía y Salud. Resumen de prensa, 2005. Bogotá, Colombia: PROFAMILIA, 2005.

37. Del Boca FK, Darkes J, Goldman MS, Smith GT. Advancing the expectancy concept via the interplay between theory and research. Alcohol Clin Exp Res 2002; 26: 926-35.

38. Oei TP, Morawska A. A cognitive model of binge drinking: the influence of alcohol expectancies and drinking refusal self-efficacy. Addict Behav. 2004; 29: 159-79.

39. Young RM, Connor JP, Ricciardelli JP, Saunders JB. The role of alcohol expectancy and drinking refusal selfefficacy beliefs in university student drinking. Alcohol Alcohol. 2006; 41: 70-5

40. Musher-Eizenman DR, Kulick AD. An alcohol expectancy-challenge prevention program for at-risk college women. Psychol Addict Behav. 2003; 17: 163-6.

41. McCarthy DM, Aarons GA, Brown SA. Educational and occupational attainment and drinking behavior: an expectancy model in young adulthood. Addiction. 2002; 97: 717-26.

42. Kline RB, Canter WA. Can educational programs affect teenage drinking? A multivariate perspective. J Drug Educ. 1994; 24: 139-49.

43. Londoño-Perez C, Vinnaccia S. Prevención en el consumo de alcohol en jóvenes universitarios: Lineamentos en el diseño de programas costo-efectivos. Psicologia y Salud 2005; 2: 241-49. 